\title{
Special issue: The future of software engineering for security and privacy
}

\author{
Shinichi HONIDEN ${ }^{1}$ and Bashar NUSEIBEH ${ }^{2}$ \\ ${ }^{1}$ National Institute of Informatics \\ ${ }^{1}$ The University of Tokyo \\ ${ }^{2}$ Department of Computing, The Open University
}

The scale of misuse of mission-critical assets manipulated by computer-based systems has increased, because of their worldwide accessibility through the Internet and the automation of systems. Security is concerned with the prevention of such misuse. The systematic development of software that considers security risks and threats explicitly is increasingly recognized as critical to improving overall systems security. This special issue provides a forum for discussing research directions in software engineering for developing secure systems more effectively. It comprises seven papers that underwent one or more cycles of anonymous peer review and revision.

The first paper on "PORTAM: Policy, Requirements and Threats Analyzer for Mobile Code Application", by Haruhiko Kaiya, Kouta Sasaki, and Kenji Kaijiri, presents an automated tool to support users and providers of information systems, to help them understand the threats and the requirements of these systems. The paper focuses on Java mobile code applications.

The second paper on "Curriculum Design and Methodologies for Security Requirements Analysis", by Kenji Taguchi and Yasuyuki Tahara, describes the authors' Security Requirements Analysis educational course, part of the Top SE project, to teach the students how to find and fix security defects in software, as early as possible in the systems development life cycle.

The third paper on "A Survey on Security Patterns", by Nobukazu Yoshioka, Hironori Washizaki, and Katsuhisa Maruyama, surveys existing approaches to establishing security patterns, which are reusable solutions to security problems, and illustrates a direction for the integration of patterns into the development life cycle.

The fourth paper on "Software Security Engineer- ing in Wireless Sensor Networks", by Eric Platon and Yuichi Sei, surveys the state of the art of security engineering in wireless sensor networks. The paper overviews security issues and solutions that are specific to this application area.

The fifth paper on "CAMNEP: An Intrusion Detection System for High-Speed Networks", by Martin Rehák, Michal Pěchouček, Karel Bartoš, Martin Grill, Pavel Čeleda, and Vojtěch Krmíček, presents the author's research that aims to detect malicious traffic in high-speed networks by means of correlated anomaly detection methods.

The sixth paper on "Feature Interaction: the Security Threat from within Software Systems", by Armstrong Nhlabatsi, Robin Laney, and Bashar Nuseibeh, reviews the literature on offline approaches to detecting feature interactions, and identifies research challenges specifically arising from feature interactions that result in security violations.

The final paper on "Placing Computer Security at the Heart of Learning", by Mike Richards, Blaine Price, and Bashar Nuseibeh, presents the educational approach adopted at the UK's Open University for teaching computer security to large numbers of students at a distance, through supported open learning.

The guest editors would like to thank the authors of papers in this special issue for their substantive contributions. These papers will be of value to researchers, educators, and practitioners, justifiably concerned about systems security. We also would like to thank the many reviewers for devoting time and effort to improve the quality of papers this issue. Finally, we would like to acknowledge the UK Engineering and Physical Research Council (EPSRC) and The Royal Society for their financial support. 


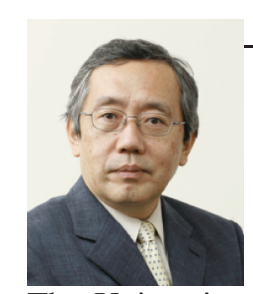

\section{Shinichi HONIDEN}

Shinichi HONIDEN is Professor and

Director of Information Systems Architecture Research Division at the National Institute of Informatics, Professor in the Graduate School of Information Science and Technology, The University of Tokyo, and a Visiting Professor at University College London and Waseda University in Japan. He received his $\mathrm{PhD}$ degree in electrical engineering from Waseda University, in 1986. From 1978 to 2000 he was with Toshiba Corporation. He was an invited professor at Le Laboratoire d'Informatique de Paris 6, Pierre et Marie Curie in 2006. His research interests include agent technology, ubiquitous computing, and software engineering. He is Chair of IEEE Computer Society Japan Chapter.

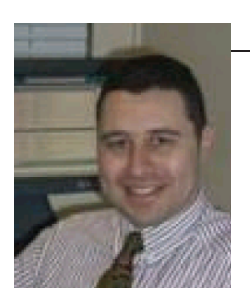

\section{Bashar NUSEIBEH}

Bashar NUSEIBEH is Professor and Director of Research in Computing at The Open University, and a Visiting Professor at Imperial College London and the National Institute of Informatics, Japan. He received his $\mathrm{PhD}$ degree in Software Engineering from Imperial College London in 1994. His research interests are in software requirements engineering and design, particularly applied to the development of dependable, missioncritical systems. Professor Nuseibeh is Editor-in-Chief of the Automated Software Engineering Journal, Chair of IFIP Working Group 2.9 on Requirements Engineering, and Chair of the Steering Committee of the International Conference on Software Engineering. He received a number of research and service awards, and is an Automated Software Engineering Fellow, a Fellow of the British Computer Society and the Institution of Engineering and Technology, and is a Chartered Engineer. 